\title{
VERSIÓN ESPAÑOLA DEL CUESTIONARIO EPQR-ABREVIADO (EPQR-A) (I): ANÁLISIS EXPLORATORIO DE LA ESTRUCTURA FACTORIAL
}

\author{
BONIFACIO SANDIN, ROSA M. VALIENTE, PALOMA CHOROT, \\ MARGARITA OLMEDO y MIGUEL A. SANTED \\ Universidad Nacional de Educación a Distancia
}

(Aceptado en agosto de 2002)

\begin{abstract}
El objetivo del presente estudio se ha centrado en analizar la estructura factorial del Eysenck Personality Questionnaire Revised-Abbreviated (EPQR-A). Francis et al. [Francis, L.J., Brown, L.B., \& Philipchalk, R. (1992). The development of an abbreviated form of the Revised Eysenck Personality Questionnaire (EPQR-A): Its use among students in England, Canada, the U.S.A and Australia. Personality and Individual Differences, $13,443-449$ ] presentaron datos preliminares sobre la fiabilidad y validez del EPQR-A. Sin embargo, aún no ha sido estudiada la estructura del cuestionario mediante análisis factorial exploratorio. En este trabajo examinamos la estructura factorial de la versión española del EPQR-A a partir de una muestra de 263 estudiantes universitarios. Aplicando análisis de componentes principales, constatamos una estructura de cuatro factores y evidencia sobre la unidimensionalidad de las cuatro subescalas del EPQRA (i.e., extraversión, neuroticismo, psicoticismo y sinceridad). Los resultados proporcionan evidencia en apoyo de la validez factorial exploratoria de la versión española del EPQR-A.
\end{abstract}

Palabras clave: EPQR-A, EPQ, extraversión, neuroticismo, psicoticismo, sinceridad, análisis factorial. Spanish version of the Eysenck Personality Questionnaire-Revised (EPQR-A) (I):
Exploratory factor analysis

The aim of this study was to examine the factor structure of the Eysenck Personality Questionnaire Revised-Abbreviated (EPQR-A). Francis et al. [Francis, L.J., Brown, L.B., \& Philipchalk, R. (1992). The development of an abbreviated form of the Revised Eysenck Personality Questionnaire (EPQR-A): Its use among students in England, Canada, the U.S.A and Australia. Personality and Individual Differences, 13, 443-449] presented preliminary data on the reliability and validity of the EPQR-A. However, the structure of the questionnaire has not been evaluated yet using exploratory factor analysis. The factor structure of the Spanish EPQR-A was examined in a sample of 263 undergraduate university students. Using principal component analysis, evidence was found for the four-factor structure and for the unidimensionality of the four EPQR-A subscales of extraversion, neuroticism, psychoticism and the lie scale. Results provide evidence for exploratory factor validity of the Spanish EPQR-A.

Key words: EPQR-A, EPQ, extraversion, neuroticism, psychoticism, lie scale, factor analysis.

\section{INTRODUCCIÓN}

El cuestionario de personalidad desarrollado por el grupo de H. J. Eysenck, no

Correspondencia: Bonifacio Sandín, Facultad de Psicología, Universidad Nacional de Educación a Distancia (UNED), Ciudad Universitaria s/n, 28040 Madrid. sólo es posiblemente el cuestionario sobre dimensiones (rasgos) básicas de la personalidad más empleado y conocido en el mundo, sino también uno de los que han sufrido mayores transformaciones. Podríamos decir que la saga de los cuestionarios de personalidad de este autor se inicia con el Maudsley Medical 
Questionnaire de 40 items (MMQ; Eysenck, 1952), y continúa con los cuestionarios Maudsley Personality Inventory de 48 items (MPI; Eysenck, 1959), Eysenck Personality Inventory de 57 items (EPI; Eysenck y Eysenck, 1964), Eysenck Personality Questionnaire de 90 items (EPQ; Eysenck y Eysenck, 1975) y, finalmente, el Eysenck Personality Questionnaire Revised de 100 items (EPQR; Eysenck, Eysenck y Barrett, 1985). Como puede apreciarse, existe una clara tendencia hacia un incremento del número de elementos del cuestionario, lo cual, al margen de una posible mejora en la fiabilidad de la prueba, también ha venido determinada por la necesidad de incrementar el número de dimensiones (concretamente por la inclusión de la dimensión de psicoticismo que se produce a partir del EPQJ.

El EPQR constituye sin duda la prueba de autoinforme para la evaluación de la personalidad más elaborada entre las desarrolladas por el grupo de Eysenck. Sin embargo, también es la prueba más larga; consta de 100 items, a partir de los cuales se evalúan las dimensiones de extraversión, neuroticismo, psicoticismo y sinceridad. Éste último aspecto confiere ciertas dificultades al EPQR, en particular si se desea obtener una valoración rápida sobre las dimensiones de la personalidad, o bien si se precisa incluir en un protocolo de múltiples pruebas de evaluación en un proyecto de investigación o en un estudio clínico. Los cuestionarios largos suelen presentar, en este sentido, importantes desventajas prácticas y, salvo que sea muy necesario, suelen evitarse en los estudios que exigen un conjunto amplio de variables a evaluar.

Por esta razón, siempre ha existido una tendencia a elaborar formas abreviadas cuando se trata de cuestionarios con un amplio número de items. Así por ejemplo, ya el propio Eysenck (1958), mediante una forma breve del MPI, evaluó la extraversión y el neuroticismo a partir de 12 items (6 items para cada dimensión). Estas dos subescalas de sólo 6 items cada una han demostrado ser válidas y fiables, y han sido aplicadas en un amplio número de estudios empíricos sobre la personalidad (Francis, Brown y Philipchalk, 1992). Por tanto, la elaboración de formas breves de cuestionarios de autoinforme no sólo es una cuestión interesante sino, sobre todo, algo útil y necesario tanto para la investigación como para la práctica clínica.

Partiendo de esta idea, Francis et al. (1992) han desarrollado una forma abreviada del EPQR (i.e., EPQR-A, Eysenck Personality Questionnaire Revised-Abbreviated) de 24 items; contiene cuatro subescalas de 6 items cada una. Las subescalas, al igual que en el EPQR, son Extraversión, Neuroticismo, Psicoticismo y Sinceridad (esta última es una medida sobre deseabilidad social, destinada a medir la tendencia a mentir al responder la prueba). Si bien es cierto que ya existía una forma abreviada del EPQR (i.e., Eysenck Personality Questionnaire Revised-Short Form, EPQR-S; Eysenck et al., 1985), esta forma aún era demasiado amplia, ya que constaba de 48 items (12 items para cada una de las cuatro subescalas).

Para la elaboración del EPQR-A, Francis et al. (1992) aplicaron a una muestra de estudiantes universitarios (procedentes de USA, Canadá, Australia e Inglaterra) el EPQ y el EPQR-S, aunque la selección de los items se llevó a cabo exclusivamente a partir de los análisis relativos al EPQR-S. Los 24 items que conforman el EPQR-A definitivo fueron seleccionados sobre la base de las correlaciones entre cada uno de los items del EPQR-S y la puntuación total en la subescala relevante (correlaciones ítem-total). Los 6 items que, para cada subescala, presentaban las mayores correlaciones ítem-total fueron retenidos para formar parte del EPQR-A. Los autores han pro- 
porcionado, así mismo, niveles apropiados sobre fiabilidad (consistencia interna) y validez convergente y divergente, si bien asumiendo que la subescala de psicoticismo presentaba niveles más bajos de fiabilidad y validez convergente; fenomeno este que ha sido corroborado mediante posteriores estudios llevados a cabo con el cuestionario (Valiente, Sandín, Chorot y Santed, 1996; Katz y Francis, 2000; Caruso, Witkiewitz, Belcourt y Gottlieb, 2001).

Particularmente nos ha llamado la atención el hecho de que el EPQR-A haya sido construido basándose únicamente en les correlaciones ítem-total (al margen, por supuesto, de otra información menos sustantiva en este sentido, como los coeficientes alfa o las correlaciones entre subescalas). Algunos estudios basados en análisis factorial exploratorio (Valiente et al., 1996) o confirmatorio (Forrest, Lewis y Shevlin, 2000; Shevlin, Bailey y Adamson, 2002) han proporcionado información adicional que confirma la consistencia de las dimensiones de personalidad extraversión y neuroticismo tal y como son evaluadas por el EPQR-A, así como la unidimensionalidad de cada una de las subescalas, lo cual constituye un apoyo empírico importante a los resultados de Francis et al. (1992). Sin embargo, de acuerdo con estos estudios, la dimensión de psicoticismo ha resultado ser poco consistente.

En un estudio piloto llevado a cabo por nuestro grupo (Valiente et al., 1996), en el que presentábamos la versión española del EPQR-A y algunos datos preliminares, constatamos factorialmente la unidimensionalidad de las cuatro subescalas del cuestionario. Observamos, no obstante, que algunos items, especialmente de la dimensión de psicoticismo (incluidos los Items 3 y 16), no saturaban de forma importante en el factor relevante. Por otra parte, la fiabilidad (consistencia interna) de las subescalas de psicoticismo y sinceridad resultaron ser bajas (en torno a 0,50 ).
Dado que, aparte de nuestro trabajo preliminar (Valiente et al., 1996), aún no se ha llevado a cabo ningún estudio exploratorio sobre la estructura factorial del EPQR-A, mediante la presente investigación pretendemos examinar directamente dicha estructura a través de la aplicación de análisis de componentes principales. Lo cual, además de poder proporcionar evidencia factorial exploratoria a favor o en contra de la estructura original del cuestionario, nos permitirá obtener información específica sobre la validez factorial de la versión española del EPQR-A. Así mismo, y con objeto de poder comparar nuestros resultados con los de Francis et al. (1992), así como con los posteriores estudios de Forrest et al. (2000) y Shevlin et al. (2002), emplearemos, al igual que estos autores, una muestra de estudiantes universitarios.

De acuerdo con la evidencia teórica y empírica sobre el EPQR-A establecemos las siguientes hipótesis: (1) El análisis de componentes principales del EPQR-A debería dar lugar a cuatro factores primarios como mejor solución factorial de la estructura simple; estos factores deberán corresponder a las dimensiones de extraversión, neuroticismo, psicoticismo y sinceridad. (2) Puesto que se asume que las dimensiones de personalidad evaluadas son relativamente independientes (no son extremos de un continuo, sino dimensiones separadas), las correlaciones entre los factores deberán ser bajas. (3) Finalmente, y de acuerdo con la anterior hipótesis, la rotación ortogonal debería reflejar la estructura de las dimensiones subyacentes mejor que la rotación oblicua.

\section{MÉTODO}

\section{Participantes}

Los cuestionarios fueron cumplimentados por una muestra total de 263 estu- 
diantes universitarios de la Universidad Nacional de Educación a Distancia. La muestra incluía 160 mujeres $(60,8 \%)$ y 103 varones $(39,2 \%)$. Ambos grupos poseían medias de edad similares; la media de edad de las mujeres era de 22,8 $(D T=7,5)$; la media de edad del grupo de hombres era de $23,2(D T=8,2)$. El rango de edad para la muestra total osciló entre 18 y 53 años.

\section{Pruebas psicológicas}

Los participantes contestaron de forma colectiva en diversos centros docentes de la UNED a los dos cuestionarios siguientes:

Cuestionario EPQR-A [Eysenck Personality Questionnaire Revised-Abbreviated] (Francis et al., 1992). Se aplicó la versión española del cuestionario (Sandín, Valiente y Chorot, 1999), la cual, como la versión original, consta de 24 items y cuatro subescalas (Extraversión, Neuroticismo, Psicoticismo y Sinceridad), correspondiendo 6 items a cada una de las subescalas. El formato de respuesta es de Sí (1) vs. No (0), con un rango de puntuaciones para cada subescala entre 0 y 6 . Las tres primeras subescalas indicadas miden rasgos de personalidad, mientras que la última evalúa la tendencia a mentir (en realidad evalúa la tendencia a emitir respuestas de deseabilidad social; respecto a esta subescala, aunque en la versión original se evalúa el nivel de mentiras, en la versión española, al igual que ocurre con el cuestionario EPQ, se evalúa inversamente, esto es, se calcula el nivel de sinceridad). (La versión española del EPQR-A se incluye en el Anexo I).

Cuestionario EPQ [Eysenck Personality Questionnaire] (Eysenck y Eysenck, 1975). Se aplicó la versión española del cuestionario (Eysenck y Eysenck, 1983).
La versión española del EPQ ha sido suficientemente validada (Eysenck y Eysenck, 1983), consta de 94 items y mide las cuatro mismas dimensinnes que el EPQR-A. El formato de respuesta es similar a este último cuestionario (Sí/No).

\section{Tratamiento estadístico}

Para examinar la estructura factorial del EPQR-A se llevaron a cabo análisis factoriales exploratorios sobre los 24 items del cuestionario, para lo cual se aplicaron análisis de componentes principales. Para la extracción del número de factores se tuvieron en cuenta los siguientes criterios teóricos y/o empíricos: (1) Modelo teórico en el que se apoya el EPQR-A, el cual sugiere la existencia de tres factores de rasgos de personalidad (extraversión, neuroticismo y psicoticismo) y un factor de sinceridad. (2) Evidencia de la literatura sobre la consistencia de estas cuatro dimensiones. (3) Criterio de Kaiser; se basa en la retención de factores con autovalores por encima de la unidad. (4) Test de scree; se basa en la representación gráfica de todos los autovalores (Cattell, 1966). (5) Finalmente, se aplic6 el criterio de interpretabilidad de las diferentes estructuras factoriales factibles (Gorsuch, 1983). La rotación ortogonal se efectuó mediante el método varimax; la rotación oblicua mediante el método oblimin. Estos son los dos tipos de rotación más utilizados, y son adecuados para los análisis factoriales exploratorios.

\section{RESULTADOS}

Análisis factorial exploratorio a partir de los items originales del EPQR-A

Tras la aplicación del análisis de componentes principales obtuvimos ocho factores con autovalores superiores a la uni- 
dad, siendo estos autovalores como sigue (entre paréntesis se indica el porcentaje de varianza explicado por cada factor): 3,60 (13,4\%), 3,19 (11,5\%), 2,65 (9,9\%), $2,00(7,2 \%), 1,59(5,9 \%), 1,43(5,4 \%)$, $1,16(4,4 \%)$, y $1,11(4,2 \%)$. La aplicación del test de scree sugería una estructura de 4 ó 5 factores, si bien los restantes criterios empíricos y/o teóricos indicaban que la mejor estructura correspondía a 4 factores; por lo cual se extrajeron 4 factores, que explicaban el $42,0 \%$ de la varianza total.

Inicialmente a estos cuatro factores se les aplicó la rotación oblicua y se obtuvieron las correlaciones entre los compo- nentes. A partir de la rotación oblicua se evidenció que el primer factor correspondía a la dimensión de sinceridad, el segundo a la dimensión de extraversión y el tercero a la dimensión de neuroticismo. El cuarto factor resultó ser más ambiguo, si bien en cierto modo parecía reflejar la dimensión de psicoticismo. Las correlaciones entre los cuatro factores resultaron ser muy bajas, ya que oscilaron entre 0,03 [correlación entre los factores segundo (extraversion) y cuarto (psicoticismo)] y $-0,13$ [correlación entre los factores primero (sinceridad) y tercero (neuroticismo)]. Por tanto, con objeto de intentar obtener una solución factorial

Tabla 1. Análisis de componentes principales: Saturaciones obtenidas para los items del EPQR-A en cada uno de los cuatro factores después de la rotación ortogonal (varimax) $(N=263)$

\begin{tabular}{|c|c|c|c|c|c|c|}
\hline ftem del EPQR-A & & travers & aroticis & icoticism & Sincerida & $h^{2}$ \\
\hline 1. ¿Tiene cambios en su estado de ánimo? & $(\mathrm{N})$ & $-0,06$ & 0,71 & $-0,01$ & $-0,07$ & 0,51 \\
\hline 2. ¿Es una persona habladora? & (E) & $\mathbf{0 , 7 4}$ & 0,14 & 0,01 & $-0,06$ & 0,57 \\
\hline 3. ¿Le preocupa tener deudas? & (P) & 0,08 & $-0,19$ & 0,18 & $-0,13$ & 0,15 \\
\hline 4. ¿Es una persona animada? & (E) & $\mathbf{0 , 5 4}$ & $-0,27$ & $-0,05$ & 0,08 & 0,37 \\
\hline 5. ¿Ha deseado ayudarse a sí mismo? & (S) & $-0,26$ & $-0,08$ & $-0,08$ & 0,41 & 0,26 \\
\hline 6. ¿Tomaría drogas? & (P) & $-0,02$ & $-0,09$ & $\mathbf{0 , 3 0}$ & 0,26 & 0,25 \\
\hline 7. ¿Ha acusado a otras personas? & (S) & 0,09 & 0,25 & 0,04 & $\mathbf{0 , 6 9}$ & 0,55 \\
\hline 8. ¿Prefiere actuar a su modo? & (P) & 0,16 & $-0,12$ & 0,28 & 0,24 & 0,25 \\
\hline 9. ¿Se siente a menudo harto? & $(\mathrm{N})$ & $-0,01$ & 0,67 & $-0,01$ & $-0,02$ & 0,46 \\
\hline 10. ¿Ha cogido algo de otra persona? & $(\mathrm{S})$ & 0,01 & $-0,05$ & 0,14 & $\mathbf{0 , 5 3}$ & 0,31 \\
\hline 11. ¿Se considera nervioso/a? & $(\mathrm{N})$ & 0,11 & $\mathbf{0 , 6 6}$ & 0,07 & $-0,16$ & 0,49 \\
\hline 12. ¿El matrimonio es algo pasado de moda? & $(\mathrm{P})$ & $-0,02$ & $-0,10$ & $\mathbf{0 , 3 1}$ & 0,18 & 0,24 \\
\hline 13. ¿Podría animar una fiesta? & (E) & $\mathbf{0 , 6 7}$ & $-0,03$ & $-0,06$ & 0,07 & 0,46 \\
\hline 14. ¿Es una persona preocupada? & $(\mathrm{N})$ & $-0,07$ & $\mathbf{0 , 5 0}$ & 0,23 & $-0,02$ & 0,37 \\
\hline 15. ¿Tiende a mantenerse en el anonimato? & (E) & 0,67 & $-0,03$ & $-0,22$ & 0,20 & 0,55 \\
\hline 16. ¿Le preocupan los errores en el trabajo? & (P) & $-0,08$ & 0,01 & $-0,15$ & 0,19 & 0,11 \\
\hline 17. ¿Ha hecho trampas jugando? & (S) & 0,07 & 0,05 & $-0,02$ & $\mathbf{0 , 6 5}$ & 0,43 \\
\hline 18. ¿Sufre de los nervios? & $(\mathrm{N})$ & 0,04 & $\mathbf{0 , 7 1}$ & 0,05 & $-0,08$ & 0,52 \\
\hline 19. ¿Se ha aprovechado de alguien? & $(\mathrm{S})$ & 0,05 & 0,06 & 0,15 & 0,76 & 0,61 \\
\hline 20. ¿Es una persona callada? & (E) & 0,84 & 0,01 & $-0,03$ & 0,01 & 0,70 \\
\hline 21. ¿Suele sentirse solo/a? & $(\mathrm{N})$ & $-0,13$ & $\mathbf{0 , 5 7}$ & $-0,08$ & 0,06 & 0,35 \\
\hline 22. ¿Es mejor seguir las normas sociales? & (P) & 0,11 & $-0,17$ & $\mathbf{0 , 3 3}$ & 0,29 & 0,26 \\
\hline 23. ¿Le consideran muy animado/a? & $(E)$ & $\mathbf{0 , 7 2}$ & $-0,03$ & 0,06 & 0,04 & 0,53 \\
\hline 24. ¿Practica siempre lo que predica? & $(\mathrm{S})$ & $-0,22$ & 0,15 & 0,21 & 0,29 & 0,25 \\
\hline
\end{tabular}

Nota: Los items se indican de forma resumida (véase el Anexo I). Entre paréntesis se indica la subescala a la que corresponde cada ftem según el cuestionario original: $\mathrm{N}=$ Neuroticismo, $\mathrm{E}=$ Extraversión, $\mathrm{P}=\mathrm{Psicoticismo}, \mathrm{S}=$ Sinceridad. En negrita se indican las saturaciones $\geq 0,30 . h^{2}=$ comunalidad. 
que delimitara de la forma más clara posible los cuatro componentes aislados, se aplicó la rotación ortogonal. Los resultados de esta rotación no mejoraron sustancialmente los obtenidos a partir de la rotación oblicua, aunque sí se modificó (el factor de extraversión pasó al primer lugar) y se delimitaron con bastante exactitud las dos principales dimensiones de la personalidad (extraversión y neuroticismo) además de la subescala de sinceridad (véase la Tabla 1).

Tal y como puede observarse a partir de la inspección de dicha tabla, las dimensiones de extraversión y neuroticismo aparecen muy bien definidas tras el análisis y parecen reflejar dos claras e independientes dimensiones de la personalidad (en ambas dimensiones las saturaciones de las variables relevantes son elevadas y las de las variables irrelevantes resultan despreciables). La dimensión de sinceridad también posee validez factorial, y se ajusta a lo que cabría esperarse tras la rotación; tal vez, la única excepción corresponde al ítem 24 , el cual no alcanza la saturación mínima esperable de 0,30 en el factor de sinceridad (no obstante, la saturación es de 0,29 , muy cercana, por tanto, a la mínima esperable). $\mathrm{El}$ factor de psicoticismo (el último en la extracción entre los cuatro componentes), sin embargo, no resulta bien definido por los datos, ya que la mitad de los items que deberían saturar en él exhiben pesos factoriales demasiado bajos (no alcanzan el valor mínimo de 0,30). En este sentido, se observan, sobre todo, las anormales saturaciones de los Items 3 y 16, así como también los bajos valores de la comunalidad para estas dos variables.

\section{Análisis factorial exploratorio del EPQR-A sustituyendo dos items originales}

Dado que la dimensión factorial de psicoticismo resultaba por lo menos pobremente definida partiendo de los datos obtenidos con los items originales del EPQR-A (Anexo I), intentamos obtener una estructura factorial más consistente sustituyendo los dos items problemáticos del EPQR-A (Items 3 y 16) por dos items del EPQ que, en principio, podrían ser más afines a la dimensión de psicoticismo del EPQRA. Para ello seleccionados los dos items de la subescala de psicoticismo del $E P Q$, no incluidos en el EPQR-A, que exhibían una mayor correlación ítemsubescala total. Estos items fueron " $i$ Lo pasaría muy mal si viese sufrir a un niño o a un animal?» y "¿Cree que la gente dedica demasiado tiempo para asegurarse el futuro mediante ahorros o seguros?».

El siguiente paso consistió en someter a análisis de componentes principales los 24 items del EPQR-A, si bien sustituyendo: (1) el f́tem 3 original ( $₫$ Le preocuparía tener deudas?") por el ítem "¿Lo pasaría muy mal si viese sufrir a un niño o a un animal?», y (2) el Ítem 16 original ( ¿Le preocupa si sabe que hay errores en su trabajo?») por el ítem «Cree que la gente dedica demasiado tiempo para asegurarse el futuro mediante ahorros o seguros?». Se obtuvieron siete factores con autovalores superiores a la unidad, siendo estos autovalores como sigue (entre paréntesis se indica el porcentaje de varianza explicado por cada factor): $3,20(13,3 \%)$, $3,16(13,1 \%), 2,42(10,1 \%), 1,57(6,6 \%)$, $1,36(5,7 \%), 1,23(5,1 \%), y=1,12(4,7 \%)$. La aplicación del test de scree y de los restantes criterios (aparte del criterio de Kaiser) sugería una estructura de 4 factores. Estos cuatro factores, que explicaban el $43,1 \%$ de la varianza total se referían, por orden, a las siguientes dimensiones del cuestionario (tras la rotación ortogonal): extraversión, sinceridad, neuroticismo y psicoticismo. 
Tabla 2. Análisis de componentes principales: Saturaciones obtenidas para los items del EPQR-A en cada uno de los cuatro factores después de la rotación ortogonal (varimax) y tras haber sido sustituidos dos items del cuestionario original $(N=263)$

\begin{tabular}{|c|c|c|c|c|c|c|}
\hline \multirow{2}{*}{$\frac{\text { Item del } E P Q R-A}{\text { 1. ¿Tiene cambios en su estado de ánimo? }}$} & \multicolumn{6}{|c|}{ Extraversión Neuroticismo Psicoticismo Sinceridad $h^{2}$} \\
\hline & $(\mathrm{N})$ & 0,04 & $\mathbf{0 , 6 0}$ & 0,21 & 0,14 & 0,53 \\
\hline 2. ¿Es una persona habladora? & (E) & 0,69 & 0,04 & $-0,03$ & $-0,07$ & 0,52 \\
\hline 3. ¿Le molesta ver sufrir a alguien? & (P) & $-0,26$ & $-0,15$ & $\mathbf{0 , 5 4}$ & 0,06 & 0,39 \\
\hline 4. ¿Es una persona animada? & (E) & 0,60 & $-0,24$ & $-0,10$ & $-0,01$ & 0,43 \\
\hline 5. ¿Ha deseado ayudarse a sí mismo? & (S) & $-0,22$ & $-0,08$ & $-0,08$ & $\mathbf{0 , 5 0}$ & 0,31 \\
\hline 6. ¿Tomaría drogas? & (P) & 0,09 & 0,03 & $\mathbf{0 , 4 9}$ & $-0,05$ & 0,25 \\
\hline 7. ¿Ha acusado a otras personas? & (S) & 0,02 & 0,17 & 0,15 & 0,66 & 0,48 \\
\hline 8. ¿Prefiere actuar a su modo? & $(\mathrm{P})$ & 0,12 & $-0,08$ & $\mathbf{0 , 5 0}$ & 0,28 & 0,35 \\
\hline 9. ¿Se siente a menudo harto? & $(\mathrm{N})$ & $-0,06$ & $\mathbf{0 , 6 2}$ & $-0,03$ & 0,14 & 0,41 \\
\hline 10. ¿Ha cogido algo de otra persona? & (S) & 0,03 & 0,01 & $-0,14$ & 0,62 & 0,41 \\
\hline 11. ¿Se considera nervioso/a? & $(\mathrm{N})$ & 0,06 & 0,62 & 0,19 & 0,05 & 0,43 \\
\hline 12. ¿El matrimonio es algo pasado de moda? & (P) & 0,06 & 0,08 & 0,67 & $-0,05$ & 0,47 \\
\hline 13. ¿Podría animar una fiesta? & (E) & $\mathbf{0 , 7 0}$ & 0,03 & 0,10 & 0,04 & 0,50 \\
\hline 14. ¿Es una persona preocupada? & $(\mathrm{N})$ & $-0,05$ & $\mathbf{0 , 6 0}$ & 0,08 & $-0,05$ & 0,37 \\
\hline 15. ¿Tiende a mantenerse en el anonimato? & (E) & $\mathbf{0 , 7 1}$ & $-0,05$ & $-0,01$ & 0,08 & 0,52 \\
\hline 16. ¿Se dedica demasiado a asegurar el futuro? & $(\mathrm{P})$ & $-0,05$ & 0,07 & $\mathbf{0 , 3 6}$ & 0,06 & 0,20 \\
\hline 17. ¿Ha hecho trampas jugando? & (S) & 0,06 & 0,01 & 0,10 & $\mathbf{0 , 6 5}$ & 0,43 \\
\hline 18. ¿Sufre de los nervios? & $(\mathrm{N})$ & 0,03 & $\mathbf{0 , 6 6}$ & $-0,20$ & 0,08 & 0,49 \\
\hline 19. ¿Se ha aprovechado de alguien? & $(\mathrm{S})$ & 0,04 & 0,13 & 0,20 & $\mathbf{0 , 7 2}$ & 0,57 \\
\hline 20. ¿Es una persona callada? & (E) & $\mathbf{0 , 8 1}$ & 0,06 & $-0,02$ & $-0,01$ & 0,66 \\
\hline 21. ¿Suele sentirse solo/a? & $(\mathrm{N})$ & $-0,05$ & $\mathbf{0 , 5 4}$ & 0,18 & $-0,01$ & 0,33 \\
\hline 22. ¿Es mejor seguir las normas sociales? & (P) & 0,05 & $-0,11$ & $\mathbf{0 , 4 3}$ & 0,23 & 0,25 \\
\hline 23. ¿Le consideran muy animado/a? & (E) & $\mathbf{0 , 7 0}$ & $-0,06$ & 0,02 & 0,02 & 0,50 \\
\hline 24. ¿Practica siempre lo que predica? & $(S)$ & $-0,02$ & 0,12 & 0,16 & $\mathbf{0 , 4 5}$ & 0,31 \\
\hline
\end{tabular}

Nota: Los items se indican de forma resumida (véase el Anexo II). Entre paréntesis se indica la subescala a la que corresponde cada ftem según el cuestionario original: $\mathrm{N}=$ Neuroticismo, $\mathrm{E}=$ Extraversión, $\mathrm{P}=\mathrm{Psicoticismo}, \mathrm{S}=$ Sinceridad. En negrita se indican las saturaciones $\geq 0,30 . h^{2}=$ comunalidad.

Aplicando la rotación ortogonal obtuvimos los resultados que presentamos en la Tabla 2. Como puede observarse, por el hecho de haber sustituido dos items del cuestionario original, la estructura ha mejorado notablemente, en particular en lo que concierne al factor de psicoticismo. Aunque esta dimensión sigue siendo la menos consistente (continúa siendo el último factor, y presenta saturaciones y comunalidades más bajas), la configuración de este factor es muy coherente ya que los seis items de psicoticismo saturan en él por encima de 0,30 y no se observan en el mismo otras saturaciones iguales o superiores a este valor. En general, pues, los datos indican una estructura factorial muy consistente con las cuatro dimensiones subyacentes; los factores están muy bien definidos, no existe ningún «ítem hiperplano», y tampoco existe ningún «ítem complejo».

La rotación oblicua proporcionó una estructura factorial semejante a la que hemos presentado en la Tabla 2. La única diferencia notoria consistió en el cambio de orden entre los dos primeros factores (primero sinceridad, segundo extraver- 
sión). Las correlaciones entre los cuatro factores fueron muy bajas (prácticamente despreciables) y oscilaron entre 0,01 [correlación entre los factores primero (sinceridad) y segundo (extraversión) y 0,14 [correlación entre los factores primero (sinceridad) y cuarto (psicoticismo)].

\section{DISCUSIÓN}

El presente trabajo ha tenido como objetivo estudiar la estructura factorial del EPQR-A. Que nosotros sepamos, aparte de nuestro trabajo preliminar (Valiente et al., 1996), hasta estos momentos aún no se había llevado a cabo ningún estudio dirigido específicamente a examinar dicha estructura. Los autores de esta forma abreviada (Francis et al., 1992) no aplicaron análisis factorial para aislar los items relevantes, ni han llevado a cabo otros estudios dirigidos a probar factorialmente las dimensiones del cuestionario. Sólo indirectamente Forrest et al. (2000) y Shevlin et al. (2002) han validado mediante análisis estructural las dimensiones de personalidad que evalúa el cuestionario. Algunos autores, no obstante, han indicado que la dimensión de psicoticismo posee escasa fiabilidad y validez (Katz y Francis, 2000; Caruso et al., 2001), incluidos los propios autores que desarrollaron el cuestionario (Francis et al., 1992).

Basándonos en los resultados que hemos obtenido a partir de nuestros análisis factoriales exploratorios sobre los items del EPQR-A quedan claras dos cosas. En primer lugar, se constata la validez factorial de las subescalas o dimensiones de extraversión, neuroticismo y sinceridad. Estas tres dimensiones son muy consistentes y reflejan bastante bien la estructura que subyace al EPQR-A, al menos en lo que concierne a las mismas. Tales resultados apoyan claramente los datos de Francis et al. (1992), así como también la evidencia referida por Forrest et al. (2000) y Shevlin et al. (2002).

En segundo lugar, nuestros datos indican que la dimensión de psicoticismo, tal y como es evaluada a partir de la versión española del EPQR-A, resulta menos consistente y peor definida que las anteriores dimensiones. Por tanto, la validez factorial de esta dimensión resulta cuanto menos cuestionable. Este resultado es congruente con los datos informados por Francis et al. (1992), Valiente et al. (1996), Katz y Francis (2000) y Caruso et al. (2001), los cuales en general afirman sobre la debilidad de esta subescala en relación con la fiabilidad (consistencia interna) y validez convergente; también es congruente con los datos referidos por Shevlin et al. (2002) basados en análisis factorial confirmatorio; estos autores concluyeron que los items del EPQR-A resultaban ser buenas medidas de los factores latentes correspondientes, excepto para la subescala de psicoticismo.

La sustitución de los dos items originales de la escala de psicoticismo (Items 3 y 16), ambos definidos por el concepto de "preocupación", que parecían influir en la debilidad de este factor, por dos nuevos items de psicoticismo procedentes del EPQ dio lugar a una estructura más coherente. Los datos del nuevo análisis de componentes principales indicaron que la dimensión (o subescala) de psicoticismo posee consistencia interna y validez factorial, aun cuando continúa siendo el último factor (i.e., el que explica menos varianza).

Resumiendo, en general nuestros resultados proporcionan apoyo empírico y transcultural a la validez factorial de la versión española del EPQR-A. La dimensión de psicoticismo, no obstante, resulta pobremente validada, lo cual está en línea con los datos proporcionados por otros autores sobre fiabilidad (consistencia interna) y validez factorial y convergente. Sustituyendo dos items de la 
subescala de psicoticismo se obtiene una configuración factorial más coherente, así como una representación más precisa y una mejor definición de la dimensión de psicoticismo.

Por tanto, y basándonos en estos resultados, no parece apropiado utilizar la dimensión de psicoticismo del EPQR-A original (Anexo I), aunque sí pueden usarse con garantía las tres restantes dimensiones. Si se desea aplicar el cuestionario completo, esto es, incluyendo la dimensión de psicoticismo, parece más adecuado utilizar la forma corregida (Anexo II). No obstante, sería deseable llevar a cabo nuevos estudios que permitiesen corroborar estos resultados.

El cuestionario EPQR-A posee un gran interés en el campo de la psicología y la psiquiatría, así como en otros campos de la ciencia, ya que nos permite medir en poco tiempo las tres dimensiones básicas de la personalidad (extraversión, neuroticismo y psicoticismo), además de una indicación sobre la tendencia a mentir que posee el individuo que cumplimenta el cuestionario. Estas tres dimensiones, aunque no son las únicas (existen otros modelos de personalidad importantes alternativos al de Eysenck), sí continúan siendo entre las dimensiones de personalidad más utilizadas en la investigación y en la práctica clínica. Por tanto, consideramos de gran interés contar con una versión española de este cuestionario que posea ciertas garantías de validez y fiabilidad.

\section{REFERENCIAS BIBLIOGRÁFICAS}

Caruso, J.C., Witkiewitz, K., Belcourt, A., y Gottlieb, J.D. (2001). Reliability of scores from the Eysenck Personality Questionnaire: A reliability generalization study. $E d u$ cational and Psychological Measurement, 61, 675-689.

Cattell, R.B. (1966). The scree test for the number of factors. Multivariate Behavioral Research, 3, 245-276.
Eysenck, H.J. (1952). The scientific study of personality. London: Routledge \& Kegan Paul.

Eysenck, H.J. (1958). A short questionnaire for the measurement of two dimensions of personality. Journal of Applied Psychology, 42, 14-17.

Eysenck, H.J. (1959). Manual of the Maudsley Personality Inventory. London: University of London Press.

Eysenck, H.J., y Eysenck, S.B.G. (1975). Manual of the Eysenck Personality Questionnaire. London: Hodder \& Stoughton.

Eysenck, H.J., y Eysenck, S.B.G. (1983). EPQ: Cuestionario de personalidad. Madrid: Técnicos Especialistas Asociados.

Eysenck, S.B.G., y Eysenck, H.J. (1964). Manual of the Eysenck Personality Inventory. London: University of London Press.

Eysenck, S.B.G., Eysenck, H.J., y Barrett, P. (1985). A revised version of the psychoticism scale. Personality and individual differences, 6, 21-29.

Forrest, S., Lewis, C.A., y Shevlin, M. (2000). Examining the factor structure and differential functioning of the Eysenck Personality Questionnaire Revised-Abbreviated. Personality and Individual Differences, 29, 579-588.

Francis, L.J., Brown, L.B., y Philipchalk, R. (1992). The development of an abbreviated form of the Revised Eysenck Personality Questionnaire (EPQR-A): Its use among students in England, Canada, the U.S.A and Australia. Personality and Individual Differences, 13, 443-449.

Gorsuch, R. (1983). Factor analysis. Hillsdale, NJ: Erlbaum.

Katz, Y.J., y Francis, L.J. (2000). Hebrew revised Eysenck Personality Questionnaire: Short form (EPQR-S) and abbreviated form (EPQR-A). Social Behavior and Personality, 28, 555-560.

Sandín, B., Valiente, R.M., y Chorot, P. (1999). Evaluación del estrés psicosocial. En B. Sandín (Ed.), El estrés psicosocial: Conceptos y consecuencias clínicas (pp. 245316). Madrid: Klinik

Shevlin, M., Bailey, F., y Adamson, G. (2002). Examining the factor structure and sources of differential functioning of the Eysenck 
Personality Questionnaire Revised-Abbreviated. Personality and Individual Differences, 32, 479-487.

Valiente, R., Sandín, B., Chorot, P., y Santed, M.A. (1996). Versión española del cuestio- nario EPQR-Abreviado: Datos preliminares con muestras españolas. Póster. XXXII Congreso de la Sociedad Española de Medicina Psicosomática. Madrid, 21-23 de noviembre.

Anexo I. Versión española del cuestionario EPQR-A (forma original)

\begin{tabular}{|c|c|c|c|}
\hline Sub-escala & Clave & Ítem & Descripción \\
\hline $\mathbf{N}$ & Sí & 1 & ¿Tiene con frecuencia subidas y bajadas de su estado de ánimo? \\
\hline $\mathbf{E}$ & Sí & 2 & ¿Es Vd. una persona habladora? \\
\hline $\mathbf{P}$ & No & 3 & ¿Le preocupa tener deudas? \\
\hline $\mathbf{E}$ & Sí & 4 & ¿Es Vd. más bien animado/a? \\
\hline$S$ & Sí & 5 & $\begin{array}{l}\text { ¿Alguna vez ha deseado más ayudarse a sí mismo/a que compartir con } \\
\text { otros? }\end{array}$ \\
\hline $\mathbf{P}$ & Sí & 6 & ¿Tomaría drogas que pudieran tener efectos desconocidos o peligrosos? \\
\hline S & Sí & 7 & $\begin{array}{l}\text { ¿Ha acusado a alguien alguna vez de hacer algo sabiendo que la culpa era } \\
\text { de Vd.? }\end{array}$ \\
\hline $\mathrm{P}$ & Sí & 8 & ¿Prefiere actuar a su modo en lugar de comportarse según las normas? \\
\hline $\mathrm{N}$ & Sí & 9 & ¿Se siente con frecuencia harto/a («hasta la coronilla»)? \\
\hline$S$ & Sí & 10 & $\begin{array}{l}\text { ¿Ha cogido alguna vez algo que perteneciese a otra persona (aunque sea un } \\
\text { broche o un bolígrafo)? }\end{array}$ \\
\hline $\mathrm{N}$ & Sí & 11 & ¿Se considera una persona nerviosa? \\
\hline $\mathbf{P}$ & Sí & 12 & ¿Piensa que el matrimonio está pasado de moda y que se debería suprimir? \\
\hline $\mathrm{E}$ & Si & 13 & ¿Podría animar fácilmente una fiesta o reunión social aburrida? \\
\hline $\mathbf{N}$ & Sí & 14 & ¿Es Vd. una persona demasiado preocupada? \\
\hline $\mathrm{E}$ & No & 15 & $\begin{array}{l}\text { ¿Tiende a mantenerse callado/o (o en un } 2^{9} \text { plano) en las reuniones o } \\
\text { encuentros sociales? }\end{array}$ \\
\hline $\mathbf{P}$ & No & 16 & ¿Le preocupa si sabe que ha cometido errores en su trabajo? \\
\hline$S$ & Si & 17 & ¿Alguna vez ha hecho trampas en el juego? \\
\hline $\mathbf{N}$ & Sí & 18 & ¿Sufre Vd. de los nervios? \\
\hline S & Sí & 19 & ¿Se ha aprovechado alguna vez de otra persona? \\
\hline $\mathbf{E}$ & No & 20 & Cuando está con otras personas, ¿es Vd. más bien callado/a? \\
\hline $\mathrm{N}$ & Sí & 21 & ¿Se siente muy solo/a con frecuencia? \\
\hline $\mathbf{P}$ & No & 22 & ¿Cree que es mejor seguir las normas de la sociedad que las suyas propias? \\
\hline E & Sí & 23 & ¿Las demás personas le consideran muy animado/a? \\
\hline $\mathbf{S}$ & No & 24 & ¿Pone en práctica siempre lo que dice? \\
\hline
\end{tabular}

() L.J. Francis, B. Brown y R. Philipchalk (1992). Versión española de B. Sandín (Sandín, Valiente y Chorot, 1999). Reproducido con permiso. 
Anexo II. Versión española del cuestionario EPQR-A (cambian los Items 3 y 16 del original)

\begin{tabular}{|c|c|c|c|}
\hline Sub-escala & Clave & Ítem & Descripción \\
\hline $\mathrm{N}$ & Sí & 1 & ¿Tiene con frecuencia subidas y bajadas de su estado de ánimo? \\
\hline $\mathrm{E}$ & Sí & 2 & ¿Es Vd. una persona habladora? \\
\hline $\mathrm{P}$ & No & 3 & ¿Lo pasaría muy mal si viese sufrir a un niño o a un animal? \\
\hline $\mathrm{E}$ & Sí & 4 & ¿Es Vd. más bien animado/a? \\
\hline $\mathrm{S}$ & Sí & 5 & $\begin{array}{l}\text { ¿Alguna vez ha deseado más ayudarse a sí mismo/a que compartir con } \\
\text { otros? }\end{array}$ \\
\hline$P$ & Sí & 6 & ¿Tomaría drogas que pudieran tener efectos desconocidos o peligrosos? \\
\hline $\mathrm{S}$ & Sí & 7 & $\begin{array}{l}\text { ¿Ha acusado a alguien alguna vez de hacer algo sabiendo que la culpa era } \\
\text { de Vd.? }\end{array}$ \\
\hline $\mathbf{P}$ & Sí & 8 & ¿Prefiere actuar a su modo en lugar de comportarse según las normas? \\
\hline $\mathrm{N}$ & Sí & 9 & ¿Se siente con frecuencia harto/a («hasta la coronilla»)? \\
\hline $\mathrm{S}$ & Sí & 10 & $\begin{array}{l}\text { ¿Ha cogido alguna vez algo que perteneciese a otra persona (aunque sea un } \\
\text { broche o un bolígrafo)? }\end{array}$ \\
\hline $\mathrm{N}$ & Sí & 11 & ¿Se considera una persona nerviosa? \\
\hline $\mathrm{P}$ & Sí & 12 & ¿Piensa que el matrimonio está pasado de moda y que se debería suprimir? \\
\hline E & Sí & 13 & ¿Podría animar fácilmente una fiesta o reunión social aburrida? \\
\hline $\mathrm{N}$ & Sí & 14 & ¿Es Vd. una persona demasiado preocupada? \\
\hline $\mathrm{E}$ & No & 15 & $\begin{array}{l}\text { ¿Tiende a mantenerse callado/o (o en un } 2^{2} \text { plano) en las reuniones o } \\
\text { encuentros sociales? }\end{array}$ \\
\hline $\mathrm{P}$ & Sí & 16 & $\begin{array}{l}\text { ¿Cree que la gente dedica demasiado tiempo para asegurarse el futuro } \\
\text { mediante ahorros o seguros? }\end{array}$ \\
\hline$S$ & Sí & 17 & ¿Alguna vez ha hecho trampas en el juego? \\
\hline $\mathrm{N}$ & Sí & 18 & ¿Sufre Vd. de los nervios? \\
\hline $\mathrm{S}$ & Sí & 19 & ¿Se ha aprovechado alguna vez de otra persona? \\
\hline $\mathrm{E}$ & No & 20 & Cuando está con otras personas, ¿es Vd. más bien callado/a? \\
\hline $\mathrm{N}$ & Si & 21 & ¿Se siente muy solo/a con frecuencia? \\
\hline $\mathbf{P}$ & No & 22 & ¿Cree que es mejor seguir las normas de la sociedad que las suyas propias? \\
\hline E & Si & 23 & ¿Las demás personas le consideran muy animado/a? \\
\hline$S$ & No & 24 & ¿Pone en práctica siempre lo que dice? \\
\hline
\end{tabular}

Modificado de la versión original de L.J. Francis, B. Brown y R. Philipchalk (1992). Versión española de B. Sandín (Sandín, Valiente y Chorot, 1999). Reproducido con permiso. 